\title{
Towards establishing the structure of liquid molecular transitions metal oxides through a joint experimental and computational investigation
}

\author{
Paul M. Forster ${ }^{1,2,3}$, Keith V. Lawler, ${ }^{1,3}$ Bradley Childs, ${ }^{2}$ Daniel Mast,,${ }^{1,2,3}$ Frederic Poineau ${ }^{1,3}$, \\ Alfred P. Sattelberger ${ }^{4}$, Kenneth R. Czerwinski ${ }^{1,2}$ \\ ${ }^{1}$ Department of Chemistry and Biochemistry, University of Nevada Las Vegas, Las Vegas, NV 89154, USA \\ ${ }^{2}$ Radiochemistry Program, University of Nevada Las Vegas, Las Vegas, NV 89154, USA \\ ${ }^{3}$ High Pressure Science and Engineering Center, University of Nevada Las Vegas, Las Vegas, NV 89154, USA \\ ${ }^{4}$ Energy Engineering and Systems Analysis Directorate, Argonne National Laboratory, Argonne, IL 60439, USA
}

Molecular metal oxides stable under ambient conditions are exceptional in metal oxide chemistry. One of the best-known examples occurs for group 7 where $\mathrm{M}_{2} \mathrm{O}_{7}$ molecules exist for $\mathrm{Mn}, \mathrm{Tc}$, and Re. In the case of $\mathrm{Tc}$, reactions of $\mathrm{Tc}_{2} \mathrm{O}_{7}$ and related species have been well-known to result in a red substance informally known as "technetium red" that has defied characterization efforts to date. We present a reexamination of the $\mathrm{M}_{2} \mathrm{O}_{7}$ class of molecular metal oxides from a joint computational and experimental approach. The $\mathrm{M}_{2} \mathrm{O}_{7}$ molecules offer some unusual chemistry, including three-center covalent bonding, negative thermal bond expansion, and (in the case of Re) a structure that is partially molecular and partially extended. Insights from our work on this family have led us to propose a likely model for "technetium red" based on an extensive computational investigation. Our model, which matches experiment closely, consists of a monomer with coordinatively unsaturated Tc sites which form strong and extensive interactions with adjacent molecules, predominantly involving Tc-Tc bonds, leading to a complex fluid. 